\title{
Criminologie
}

\section{Le traitement de la toxicomanie comme alternative à l'incarcération : un sentier rocailleux}

\section{Pascal Schneeberger et Serge Brochu}

Volume 33, numéro 2, automne 2000

Problèmes sociaux et système pénal

URI : https://id.erudit.org/iderudit/004745ar

DOI : https://doi.org/10.7202/004745ar

Aller au sommaire du numéro

\section{Éditeur(s)}

Les Presses de l'Université de Montréal

ISSN

0316-0041 (imprimé)

1492-1367 (numérique)

Découvrir la revue

Citer cet article

Schneeberger, P. \& Brochu, S. (2000). Le traitement de la toxicomanie comme alternative à l'incarcération : un sentier rocailleux. Criminologie, 33(2),

129-149. https://doi.org/10.7202/004745ar
Résumé de l'article

Toxicomanie et criminalité sont des comportements fréquemment associés. Dans un objectif de réduction de la récidive, le traitement des toxicomanes apparaît dans la littérature américaine comme une solution plus efficace que l'incarcération et beaucoup moins dispendieuse. Qu'en est-il au Québec ? Pour répondre à cette question, 20 intervenants de la région de Montréal et de Trois-Rivières œuvrant dans le domaine de la justice, de la sécurité publique et de la santé et des services sociaux ont été rencontrés dans le cadre de groupes de discussion. Les résultats de la consultation montrent que si le traitement des toxicomanes judiciarisés est une idée intéressante en soi, plusieurs difficultés surgissent dans son application en raison notamment de la différence de culture des milieux impliqués. Cependant, une bonne communication, le respect des expertises de chacun, et une certaine tolérance vis-à-vis des objectifs de ses partenaires constituent des conditions qui rendraient possible, du moins sur papier, les expériences de déjudiciarisation en matière de toxicomanie. 


\title{
Le traitement de la toxicomanie comme alternative à l'incarcération : un sentier rocailleux ${ }^{1}$
}

\author{
Pascal Schneeberger \\ Agent de recherche \\ Centre international de criminologie comparée \\ Université de Montréal \\ schneebp@ere.umontreal.ca \\ Serge Brochu \\ Professeur et directeur \\ Centre international de criminologie comparée \\ Université de Montréal \\ brochus@cicc.umontreal.ca
}

RÉSUMÉ - Toxicomanie et criminalité sont des comportements fréquemment associés. Dans un objectif de réduction de la récidive, le traitement des toxicomanes apparaît dans la littérature américaine comme une solution plus efficace que l'incarcération et beaucoup moins dispendieuse. Qu'en est-il au Québec ? Pour répondre à cette question, 20 intervenants de la région de Montréal et de Trois-Rivières œuvrant dans le domaine de la justice, de la sécurité publique et de la santé et des services sociaux ont été rencontrés dans le cadre de groupes de discussion. Les résultats de la consultation montrent que si le traitement des toxicomanes judiciarisés est une idée intéressante en soi, plusieurs difficultés surgissent dans son application en raison notamment de la différence de culture des milieux impliqués. Cependant, une bonne communication, le respect des expertises de chacun, et une certaine tolérance vis-à-vis des objectifs de ses partenaires constituent des conditions qui rendraient possible, du moins sur papier, les expériences de déjudiciarisation en matière de toxicomanie.

1. Les auteurs désirent remercier le Comité permanent de lutte à la toxicomanie pour leur support relatif au déroulement de cette étude.

Criminologie, vol. 33, $n^{\circ} 2$ (2000) 
ABSTRACT - The relation between substance abuse and criminality is well demonstrated. American scientific papers tend to indicate that treatment of substance abusers seems to be a good way to reduce repeat offences at lower costs than incarceration. What is the situation in Quebec? To answer this question, 20 professionals working in Montreal and Trois-Rivières, and associated with the Department of Justice, Public Security and Health and Social Services, have been interviewed. The results show that many difficulties are encountered in part because of cultural differences between professionals. However, good communication, respect of everyone's expertise and a certain tolerance toward partners' objectives create conditions that allows, at least on paper, non judiciary intervention in substance abuse matters.

\section{Problématique}

L'existence d'une relation entre la consommation de certaines substances psychoactives (habituellement les plus dispendieuses et les plus asservissantes) et quelques formes de criminalité (surtout lucratives) chez un certain nombre d'individus (les personnes dépendantes, sans le sou, ou déjà impliquées dans la criminalité) ne fait maintenant plus de doute. En effet, de nombreux auteurs ont analysé le phénomène à partir de données empiriques, de récits de vie et d'observations ethnographiques et, même s'ils en arrivent parfois à des explications relativement différentes, ils n'en perçoivent pas moins une certaine proximité entre les deux comportements (voir Brochu, 1995, pour une recension des écrits).

En raison des activités délictueuses commises par certains consommateurs de substances psychoactives, on retrouve fréquemment cette clientèle dans les filets de la justice pénale. À titre d'exemple, Mumola (1998) mentionne que $51 \%$ des détenus américains de son échantillon rapportent avoir été sous l'influence de l'alcool ou de la drogue lorsqu'ils ont commis le crime pour lequel ils sont incarcérés. Au Canada, une récente étude indique que le quart des prisonniers interrogés avouent que la plupart de leurs crimes ont été commis sous l'effet de la drogue ou de l'alcool (Brochu et al., sous presse). Enfin, les études de prévalence indiquent généralement qu'entre le quart et la moitié des détenus canadiens seraient aux prises avec des problèmes d'alcoolisme ou de toxicomanie (Brochu, 1995).

Les intervenants des systèmes judiciaires et correctionnels n'ont pas tardé à réaliser que le simple enfermement de ces individus n'est pas suffisant pour régler les problèmes de consommation et garantir la réadaptation et que, au contraire, on ne peut penser mettre un terme à la criminalité de ces personnes sans aborder les problèmes de surconsom- 
mation et de toxicomanie qui accompagnent cette délinquance, postulant ainsi que l'arrêt de la consommation entraînera la cessation de la criminalité (Ball et al., 1981). C'est à partir de ce principe que différentes initiatives ont été élaborées et appliquées auprès des toxicomanes judiciarisés. Lorsqu'on les examine, on remarque que plusieurs formules ont été tentées, tant en ce qui concerne la modalité du traitement offert, que du type d'intervenant dispensant l'intervention et du moment auquel cette intervention s'inscrit sur le continuum de la prise en charge pénale.

Il va de soi que les différentes instances gouvernementales ont porté une attention particulière à l'évaluation des diverses mesures adoptées en la matière. Un certain nombre d'études visant à mesurer l'efficacité des programmes de traitement auprès d'une clientèle judiciarisée ont démontré un pronostic plutôt sombre quant à l'impact du traitement sur ces personnes (Steer, 1980; Simpson et al., 1986; Anglin et Hser, 1990; Beaudet et al., 1995). Ce mauvais pronostic serait attribuable à la faible motivation de cette clientèle à poursuivre une démarche volontaire de réadaptation suffisamment longtemps pour en retirer de véritables bénéfices (Beaudet et al., 1995). Toutefois, d'autres études indiquent que lorsqu'on réussit à maintenir ces individus en traitement, le pronostic s'améliore nettement (Anglin et al., 1990), laissant penser que le succès de l'intervention est relié à la persévérance des clients dans un programme thérapeutique (De Leon, 1988).

Il existe différentes stratégies pour améliorer la persistance en traitement des personnes judiciarisées. La façon la plus courante que l'on retrouve dans la documentation scientifique américaine actuelle consiste à imposer des pressions légales (spectre d'une longue sentence, d'un retour en milieu carcéral, etc.). On espère ainsi que la contrainte légale s'avérera une pression assez efficace pour maintenir les personnes judiciarisées en traitement. Certaines études évaluatives effectuées aux États-Unis sur l'efficacité de cette stratégie montrent des résultats positifs en termes de rétention en traitement et d'impact du processus de réadaptation sur la rechute et la récidive (Hubbard et al., 1988; Anglin et Hser, 1990; Brecht et al., 1993; Van Stelle et al., 1994; Torres, 1996; Schwartz et Schwartz, 1998).

Toutefois, le contexte socio-politique américain differe sensiblement de celui du Canada et du Québec et on peut émettre l'hypothèse que cette différence joue un rôle quant à l'utilisation et l'efficacité des pressions judiciaires dans le traitement de la toxicomanie. L'objectif de cette étude consiste alors à mieux comprendre l'impact des contraintes judiciaires sur la prestation des services de réadaptation en toxicomanie. 


\section{Considérations méthodologiques}

L'étude a été commandée par le Comité permanent de lutte à la toxicomanie (CPLT) dont le mandat principal est de conseiller la ministre de la Santé et des Services sociaux de même que le ministre délégué à la Santé, aux Services sociaux et à la Protection de la jeunesse sur les orientations, les priorités d'action et les domaines d'intervention à privilégier en matière de toxicomanie au Québec. Réalisée en 1999, l'étude avait pour objectif de recueillir le point de vue des intervenants québécois impliqués auprès des toxicomanes judiciarisés que ce soit à titre de professionnels référant ou d'intervenants assurant la réhabilitation. Nous avons estimé que l'utilisation de groupes de discussion (focus groups) constituerait une méthode appropriée à l'objectif que nous visions. En effet, cette méthode offre l'avantage, outre d'exiger des frais de déplacement et de temps d'entrevue beaucoup moins élevés, de pousser la réflexion de chaque participant plus loin qu'il ne l'aurait fait s'il avait été rencontré individuellement. Cette méthode favorise aussi l'établissement d'un portrait global, l'ensemble du continuum étant représenté par différents acteurs autour de la même table.

Compte tenu que nos travaux dans le domaine de l'intervention en toxicomanie auprès des personnes judiciarisées ont impliqué depuis quelques années déjà des intervenants provenant de la région de Montréal, de Trois-Rivières et de la Montérégie (Brochu et Schneeberger, 1997; Brochu et al., 1999 a et b) nous avons décidé de faire appel encore une fois à leurs services. Nous avons estimé que la tenue de quatre groupes de discussion permettrait de rencontrer un nombre suffisant d'intervenants. Ainsi, trois groupes ont été constitués dans la région de Montréal (les intervenants de la Montérégie pouvant facilement s'y rendre) et un autre dans la région trifluvienne.

Pour rejoindre les participants, nous avons adopté une stratégie en deux volets. Dans un premier temps, une lettre a été envoyée à des intervenants identifiés comme jouant un rôle important dans la dynamique étudiée. Par la suite, ces personnes ont été rejointes par téléphone pour leur exposer plus en détail la nature du projet et confirmer leur présence à un des groupes de discussion.

En raison de la période estivale durant laquelle se sont déroulés les groupes de discussion, le taux de réponse à l'invitation lancée a été relativement faible. En effet, un total de 20 personnes ( 9 femmes et 11 hommes) se sont présentées à l'un des groupes de discussion alors que nous avions procédé à l'envoi de 57 lettres. Il faut dire que des lettres ont 
parfois été envoyées à plusieurs individus d'un même organisme qui ont habituellement choisi de ne déléguer qu'un seul représentant. Les 20 personnes présentes proviennent soit d'un centre de réadaptation public (7) ou privé (3) en toxicomanie, d'un service de police (3), d'une maison de transition (4) ou d'un établissement de détention (3). Chacune d'elles possède une expérience directe en intervention bien que $20 \%$ occupent actuellement un poste de cadre (directeur clinique).

Tous les groupes de discussion se sont tenus entre le 10 juin 1999 et le 23 juin de la même année et ont duré de deux heures et demie à trois heures, selon le groupe. Chaque intervenant n'était rencontré qu'une fois. Le chercheur senior a par ailleurs assuré l'animation des rencontres qui se sont toutes déroulées sensiblement de la même façon. Après avoir donné la chance à tous les participants de se présenter et d'indiquer la nature de leur travail, nous avons posé tour à tour les questions suivantes. Comment fonctionne la référence judiciaire dans votre secteur? Quels sont les types et les sources de pressions judiciaires exercés? Quelles sont les principales difficultés rencontrées ? Peut-on penser avoir un impact auprès des toxicomanes lorsqu'il y a référence du système judiciaire? Dans quelles conditions? Chacune des rencontres a été enregistrée avec l'accord des participants. Ce sont d'ailleurs ces enregistrements qui ont constitué le matériel de base de l'analyse du discours des participants puisqu'ils ont servi à la rédaction d'un compte rendu de chacune des rencontres. À la fin de chaque groupe, les participants étaient informés qu'ils recevraient le compte rendu des propos tenus lors de la discussion de manière à s'assurer que nous avions bien compris leurs propos. Ils étaient invités à nous contacter si certaines nuances ou corrections devaient être apportées à leur discours, procédure qui a été utilisée une fois par un participant qui tenait à expliciter davantage son idée. À partir des comptes rendus ainsi rédigés, l'équipe de recherche a procédé à l'analyse des rencontres en dégageant les principaux thèmes abordés par les participants. La section suivante présente les résultats de cette analyse.

\section{Analyse du contenu des groupes de discussion}

L'intervention en toxicomanie dans un contexte judiciaire peut s'avérer complexe. En effet, la double problématique toxicomanie-délinquance, son évaluation et sa prise en charge impliquent une multitude d'acteurs provenant de différents milieux. Lorsqu'un individu est appréhendé par 
la justice, il doit généralement traverser différentes étapes du processus judiciaire (arrestation par la police, enquête préliminaire, procès, condamnation, etc.). Il arrive aussi que des individus se trouvant dans l'appareil judiciaire et présentant des besoins spécifiques se voient référés à d'autres sphères de compétences. Il n'est donc pas rare que l'individu qui présente une problématique de toxicomanie soit référé à une ressource spécialisée dans la réadaptation des toxicomanes. Cette référence ou renvoi peut provenir de différentes sources.

\section{Sources de renvoi}

Les premières personnes généralement appelées à intervenir auprès des toxicomanes judiciarisés sont habituellement les policiers. Dès cette étape, la personne présentant des problèmes de toxicomanie peut être référée à une ressource pour toxicomanes. Cela ne signifie pas forcément l'interruption du processus judiciaire, quoique certains policiers puissent utiliser leur pouvoir discrétionnaire afin de référer l'individu, plutôt que de l'arrêter et d'entreprendre des poursuites. En juillet 1996, une initiative du genre avait été prise par le Service de police de la Communauté urbaine de Montréal (SPCUM) du centre-ville, en collaboration avec Portage, un centre de réadaptation privé pour toxicomanes. Il s'agissait du programme Option-toxicomanie. L'un des participants nous a expliqué que les individus arrêtés par les policiers pouvaient, à l'époque, bénéficier d'une rencontre avec un intervenant en toxicomanie et, selon leur volonté et leurs besoins, se voir référés en thérapie.

On s'est rendu compte que $50 \%$ des personnes qui ont été rencontrées ont accepté de faire un traitement. Et moi, ce que je disais quand on a commencé ça, c'est que le gars qui est assis dans une cellule en bon français il est dans la merde un peu. Si tu l'avais rencontré six mois avant et que tu lui avais dit si tu continues à consommer tu vas te retrouver un moment donné à faire des délits pour ta consommation, tu vas avoir perdu ta job, une espèce de dégringolade. Souvent les gens nous disent «Ben non je contrôle bien ma consommation, je me rendrai pas là. » Mais quand on le prend, il est rendu là. Alors à ce moment-là, c'était facile.

L'arrestation constitue, pour un bon nombre de toxicomanes, un point tournant, un moment important de leur trajectoire qui les motive à entreprendre un changement de leur mode de vie. L'intervention de déjudiciarisation et de renvoi vers un centre de traitement spécialisé préconisée par Option-toxicomanie semblait apparaître à un bon moment. Bien que la formule ait reçu un bon accueil de la part des 
personnes mises en arrestation, cette pratique a dû être interrompue après un an, faute de moyens financiers.

En second lieu, les avocats peuvent adresser une demande à la cour afin que leur client soit référé à une ressource pour toxicomanes. Cependant, certains participants des groupes de discussion rapportent qu'une telle mesure a parfois des effets pervers et peut être perçue davantage comme une façon d'alléger les sanctions pénales plutôt qu'un moyen pour modifier sa trajectoire de consommation.

Je pense qu'il y a une partie aussi qui est encouragée par les avocats. Dans le sens où les avocats avant même d'aller en cour vont dire « bon écoute va faire une démarche ça va jouer en ta faveur » qui vont faire reporter la cause, etc, etc. C'est une façon d'utiliser le système qui est un peu endossée par les juges aussi. Et je pense que les juges sont peutêtre mésinformés ou ont des croyances magiques et ils se disent : bon il y est allé, il a commencé une démarche, on lui donnera pas la même sentence compte tenu de ça, donc c'est quelque chose qui est renforcé par les juges parce que ça fonctionne. Ça fait que les avocats ont tout intérêt à l'utiliser pour le bien-être de leurs clients.

Comme on peut le voir, le souci de certains avocats de la défense semble parfois être la réduction de la peine au maximum plutôt que l'évaluation de la nécessité d'une intervention en toxicomanie.

Troisièmement, les procureurs de la couronne constituent des intervenants pénaux susceptibles d'utiliser le renvoi auprès des toxicomanescontrevenants. Généralement, ils demanderont au juge de jumeler cette mesure à une sentence suspendue, à une ordonnance de probation voire à une mise en liberté sous condition.

Quatrièmement, le renvoi à une ressource peut se réaliser par le biais des établissements de détention. Dans le cas d'un individu incarcéré dans une prison provinciale au Québec, la peine purgée est, dans la majorité des cas, inférieure à six mois. Pour cette raison, les intervenants de ces établissements croient qu'une démarche thérapeutique peut difficilement être entreprise à l'intérieur des murs. C'est pourquoi un individu présentant une problématique de toxicomanie sera référé à une ressource spécialisée au moment de sa mise en liberté sous condition. Toutefois, la Commission québécoise des libérations conditionnelles impose certaines directives à l'individu libéré afin qu'il puisse purger le reste de la peine dans la communauté. Parmi ces conditions, on retrouve souvent la condition de non-consommation et la Commission va parfois jusqu'à exiger que l'individu suive un traitement afin de régler ses problèmes de toxicomanie. 
Enfin, le Service correctionnel du Canada a mis sur pied un ensemble de programmes d'intervention en toxicomanie disponible à divers moments du cheminement carcéral. Ce dernier n'a donc généralement pas besoin de référer un individu aux prises avec des difficultés de consommation à un organisme extérieur mais l'oriente à travers les services disponibles au sein de l'organisation. Ces services couvrent à la fois la prévention et la réadaptation. Au Québec, il existe un programme pré-libératoire pour toxicomanes (PPT) qui est offert dans plusieurs pénitenciers. Ce programme est axé sur l'éducation et la réflexion en matière d'alcool et de drogues, les habiletés de résolution de problèmes, et un effort particulier est mis sur la prévention de la rechute. De plus, certains pénitenciers québécois offrent des programmes d'intervention tertiaire de forte intensité sous la forme de communautés thérapeutiques dont la durée de thérapie varie de neuf à douze mois : les programmes ÉCHO et STOP. Le premier est offert à l'établissement Leclerc, une institution à sécurité moyenne, alors que le second se retrouve au Centre fédéral de formation, un pénitencier à sécurité minimale.

\section{Le contrevenant : un toxicomane difficile à évaluer}

La question de la toxicomanie étant très présente lorsqu'on évalue les problématiques associées à la commission d'un délit, plusieurs entités du système judiciaire et de la sécurité publique se déclarent préoccupées par la question de l'évaluation des délinquants toxicomanes.

Les participants s'accordent pour affirmer qu'une bonne évaluation de la problématique toxicomane constitue la base d'un traitement efficace. Pour ce faire, il est essentiel d'examiner attentivement quatre composantes avec le contrevenant : 1) sa gestion de la consommation de substances psychoactives (p. ex. : type de substances consommées; mode de consommation; indice quantité/fréquence; niveau de dépendance, etc.); 2) la place de la drogue dans la commission de ses délits (p. ex. : la drogue estelle utilisée pour se donner du courage ? s'agit-il plutôt d'une criminalité visant à obtenir l'argent nécessaire pour se procurer sa drogue ?); 3) les sphères de sa vie pouvant être affectées par la consommation, de façon à effectuer un renvoi adéquat vers une ressource susceptible de répondre aux besoins du client (p. ex : conséquences familiales, sociales, psychologiques, et médicales de la consommation); 4) la trajectoire judiciaire de l'individu, puisque l'étape du cheminement où se trouve la personne évaluée pourrait avoir un impact considérable sur un éventuel traitement 
(p. ex. : début ou fin d'une carrière criminelle; gloire criminelle vs saturation délinquante; stades de motivation, etc.).

Si quelqu'un n'avait jamais été judiciarisé je me dirais oui ça peut avoir la même portée parce que c'est pas quelqu'un qui va avoir un profil plus haut. Mais si c'est quelqu'un qui a été judiciarisé à maintes reprises, qui a un profil délinquant, on fait face à une dynamique qui est particulière pis on peut pas faire abstraction de ça parce que ça soulève en soi des problèmes dans la relation d'aide compte tenu de leur dynamique. Et quand on parlait d'âge, ça me fait penser aussi à la courbe d'âge de la délinquance et on le voit en toxicomanie parce que quand ils passent le 35 [ans]. À partir de 30 [ans], dépendamment du monde, ça peut être 30,35, 40 [ans] y'a un moment donné où ils ne veulent plus retourner en dedans, ils sont brûlés, ils ont fait deux trois fois; et leur motivation va être différente même si leur feuille de route est lourde...

Y'a des gens avec un long passé de prison qui disent : là je suis tanné, si je retourne là moi je me pends, je me suicide, n'importe quoi, qui disent : moi je ne veux plus y aller, je m'organise pour ne plus y aller.

Comme nous l'avons vu précédemment dans l'initiative de la SPCUM et du Portage, des efforts ont été faits, dans certains cas, pour que l'évaluation du contrevenant soit réalisée le plus rapidement possible lorsqu'un individu est appréhendé par la justice. Toutefois, ces initiatives sont dispendieuses en temps et en argent. Elles requièrent la présence d'un personnel formé à l'évaluation de la problématique toxicomane. Aussi, en contexte de contraintes budgétaires, il n'est pas toujours possible de soutenir et de maintenir ce genre d'initiative.

Cette évaluation des toxicomanes judiciarisés jugée nécessaire par l'ensemble des intervenants semble cependant soulever la controverse, car elle présente, aux dires des participants de l'étude, des lacunes majeures. L'un des problèmes rencontrés s'avère le manque de formation et d'expertise en matière de toxicomanie de certains intervenants pénaux. Cette réalité occasionne parfois un renvoi inapproprié à une ressource pour toxicomanes. À cet effet, plusieurs participants remarquent qu'il existe une différence importante entre consommation et toxicomanie puisqu'on ne parle pas de dépendance dans le premier cas et que les besoins des individus different nettement d'une situation à l'autre. Or, certains intervenants pénaux à qui l'on confie la tâche d'évaluer et de préparer l'individu, ne sont pas toujours en mesure de faire ce genre de distinction étant donné le contexte illégal de la consommation de drogues. 
Moi je pense qu'il faut être capable de regarder ça [l'évaluation du toxicomane]. Mais moi je pense pas que c'est une juge qui est capable de regarder ça et c'est sûrement pas un avocat qui va être capable de regarder ça.

L'évaluation des toxicomanes judiciarisés ne devrait pas, selon les participants, s'effectuer uniquement par le personnel du réseau de la justice, mais plutôt se faire conjointement avec les experts du réseau de la toxicomanie, de façon à tenir compte des deux expertises nécessaires à une évaluation complète de la problématique du contrevenant qui abuse de drogues. Ainsi, les problématiques seraient mieux identifiées et les ressources mieux adaptées aux besoins des contrevenants.

Le traitement: une alternative assaisonnée de plusieurs conditions

Lorsqu'un individu entreprend une démarche de traitement et qu'il est pris en charge par un programme pour toxicomanes, certaines conditions se doivent d'être respectées de façon à assurer le bon fonctionnement des opérations. Cette réalité est d'autant plus vraie chez les contrevenants qui doivent rendre des comptes au système judiciaire. Plusieurs types de conditions peuvent être imposés à l'individu par les différents paliers de l'appareil pénal. De façon générale, ces conditions vont dans le sens d'un contrôle relativement rigide. À cet effet, l'un des participants rapporte que les détenus inscrits à un programme de maintien à la méthadone sont souvent doublement surveillés par le personnel de l'établissement où ils se trouvent. En effet, ils doivent prendre leur dose de méthadone telle que prescrite, mais ils doivent aussi régulièrement rendre des comptes (par exemple, la passation de tests d'urine afin de confirmer leur sobriété). Par ailleurs, un autre participant mentionne que les résidents des maisons de transition suivant un traitement sont aussi très surveillés et ne doivent commettre aucun manquement, sans quoi, ils risquent d'être incarcérés à nouveau.

Ça c'est des gens qui ont trop de pression [détenus sous méthadone] quand ils sortent de détention... au niveau des interdits légaux et ces choses-là. [Ils sont plus surveillés ou quoi ?] Ah ! ils sont surveillés, je dirais c'est presque inhumain. C'est un peu de notre faute aussi, on en met aussi des conditions. C'est des gens qui... qui ont l'obligation des tests pipi, ils ont l'obligation de la prendre la méthadone. Si une journée ils la prennent pas ça pourrait être un motif de retour en détention. C'est très encadré la prise de méthadone. Si c'est à l'hôpital juif $t$ 'as des suivis psychosociaux avec ça, ça finit plus, la recherche 
d'emploi à travers ça pis éventuellement une thérapie à DollardCormier. C'est inhumain.

Le traitement du toxicomane, qui se veut un allègement de peine, ne deviendrait-il pas son contraire par le nombre de conditions à respecter et la surveillance qui y est associée?

Outre ces conditions, les intervenants pénaux vont parfois imposer aux contrevenants la fréquentation de certaines ressources pour toxicomanes, ce qui n'est pas sans poser quelques problèmes, puisque ce ne sont pas toutes les ressources de réadaptation qui désirent intervenir dans ces conditions. En fait, c'est surtout le secteur public de réadaptation en toxicomanie qui considère d'un mauvais œil l'identification d'une ressource en particulier, prétextant que cette procédure appelle une obligation de résultats sans quoi l'individu risque une réincarcération. De plus, les intervenants des ressources publiques de réadaptation en toxicomanie ne désirent pas endosser la responsabilité de ce nouvel emprisonnement. Quant aux ressources privées, elles n'envisagent généralement pas la situation de la même façon. En effet, plusieurs d'entre elles vont même jusqu'à engager un agent de liaison qui entretiendra des contacts étroits avec les intervenants pénaux tout en leur suggérant de leur confier le traitement de tel ou tel client.

Mentionnons, par ailleurs, qu'il n'est pas rare que les intervenants pénaux exigent que leur clientèle soit accueillie dans une ressource pouvant offrir un encadrement thérapeutique en milieu résidentiel parce qu'ils accordent plus de crédibilité à ces ressources, notamment pour leur capacité de surveiller les contrevenants. La réadaptation ressemble ainsi davantage à l'incarcération.

Comme il fut mentionné précédemment, une des conditions intrinsèques au traitement des toxicomanes-contrevenants est la condition de non-consommation. Cette condition est largement répandue et utilisée par les intervenants pénaux. Toutefois, elle soulève la controverse auprès des intervenants du secteur de la réadaptation. En effet, les intervenants pénaux considèrent la rechute comme un prédicteur important d'une récidive imminente alors que les intervenants du secteur de la réadaptation sont convaincus que la rechute constitue une étape normale dans un processus de réadaptation et qu'elle offre aussi un matériel d'intervention de premier choix.

Mais s'il rechute, moi ça devient du matériel de première main pis là je lui dis qu'est-ce qui s'est passé, qu'est-ce que tu vivais à ce moment-là, 
comment tu t'es senti, le recentrer là-dessus. Pis moi me priver de ça dans un cadre de travail en toxico...

Ca peut être par exemple "démontrer la prise en charge de sa toxicomanie ». C'est pas parce que tu as eu une rechute que tu démontres pas que tu veux t'en sortir. Les rechutes font partie du cheminement [...], donc on doit accepter que les rechutes font partie de la game.

Comme on peut le constater, deux priorités et deux philosophies de l'intervention s'opposent ici. Les intervenants pénaux, en voulant protéger la société d'une récidive possible, sont accusés de priver l'intervenant en réadaptation d'un matériel clinique précieux que constituent la réflexion et le travail sur les événements qui ont précipité la rechute. Les intervenants du secteur de réadaptation apparaissent, aux yeux des agents pénaux, comme laxistes dans leur façon d'opérer les contrôles nécessaires à la sécurité de la population.

On remarque qu'il se crée autour de cette question de la condition de non-consommation une espèce de jeu, une chasse aux sorcières où les énergies des intervenants pénaux sont consacrées à l'identification de la rechute et celles des contrevenants à l'élaboration de stratégies qui permettront de déjouer les méthodes mises de l'avant par les systèmes correctionnels et judiciaires. Cette situation ne favorise certes pas le dévoilement de soi du contrevenant envers le personnel chargé de le surveiller.

Lorsque la surveillance parvient à démontrer que la condition de non-consommation n'est pas respectée par un individu en traitement, ce dernier devra, de toute évidence, en subir les conséquences. Comme nous avons pu le constater, les intervenants pénaux considèrent généralement la rechute du contrevenant non seulement comme un bris de condition, mais comme un sérieux indice d'une récidive probable, suggérant dans plusieurs cas la suspension de leur liberté et, conséquemment, la rupture avec une ressource d'aide.

\section{Punition et réadaptation : une complicité difficile}

Les difficultés liées à l'intervention en matière de toxicomanie chez une clientèle judiciarisée sont nombreuses. Ces difficultés peuvent être présentes à différents niveaux. D'abord, nous l'avons mentionné à plus d'une reprise, plusieurs intervenants pénaux ne se sentent pas en mesure de faire une évaluation adéquate des problématiques reliées à la toxicomanie des individus. Par exemple, on utilise souvent à tort l'appellation toxicomane, 
et on appose parfois trop facilement cette étiquette aux dires de certains participants. En effet, selon un participant, on ne semble pas prendre les mêmes précautions dans l'usage de ce diagnostic comme on le fait avec l'usage de la pédophilie ou de la schizophrénie. L'individu ainsi étiqueté risque fort d'être stigmatisé par la suite, même s'il ne présente pas réellement de problématique de toxicomanie. On a parfois tenté de remédier à cette situation en instaurant des mesures pour que l'évaluation se fasse par un personnel spécialisé, mais force est de constater que cette pratique n'est pas aussi répandue qu'on le souhaiterait.

De plus, la référence vers des services adéquats dans le domaine de la toxicomanie ne va pas de soi. Le ministère de la Santé et des Services sociaux n'a pas encore mis en place, au moment d'écrire ces lignes, des procédures d'accréditation des ressources en toxicomanie. La réputation des ressources s'appuie donc davantage sur de la publicité de bouche à oreille ou sur des références bien réussies que sur une évaluation réelle de leur capacité à offrir un service adéquat aux personnes référées. Bien plus, lorsqu'elles existent, les procédures à suivre pour référer un individu à une ressource sont parfois ardues et le nombre de places disponibles pour recevoir la clientèle est limité (notamment pour la clientèle féminine). Il n'est donc pas rare qu'un intervenant abandonne sa démarche de référence devant les difficultés qu'il rencontre.

La durée du mandat judiciaire constitue également une difficulté majeure chez les intervenants pénaux. Par exemple, un participant nous informe que la durée moyenne d'incarcération dans un établissement provincial est de deux mois et demi à trois mois. Or, ce laps de temps s'avère, selon lui, beaucoup trop court pour entreprendre une démarche thérapeutique, compte tenu du contexte actuel d'incarcération dans les prisons provinciales. Cela fait en sorte que peu d'initiatives (mis à part la référence à des ressources extérieures) sont mises en application pour venir en aide à la clientèle toxicomane durant son incarcération.

Les pressions judiciaires exercées chez l'individu en traitement, ainsi que sur les ressources qui les reçoivent, sont aussi au nombre des difficultés mentionnées par les participants. Le système de justice oblige en quelque sorte certains délinquants toxicomanes à suivre un traitement. Dans ces circonstances, la décision d'entreprendre une démarche thérapeutique ne provient pas de l'individu lui-même, mais de l'appareil pénal. Cette situation fait en sorte que les ressources de réadaptation reçoivent souvent des individus peu motivés à suivre un traitement, ce qui ne facilite en rien leur pratique. En revanche, la majorité des inter- 
venants des ressources de réadaptation en toxicomanie s'accordent pour dire qu'ils acceptent de travailler avec ces individus peu motivés. Leur rôle consiste par la suite à permettre aux clients d'intérioriser cette motivation extrinsèque. Toutefois, il faut bien être conscient que l'intervention en toxicomanie dans un contexte judiciaire s'effectue dans un cadre très rigide où l'individu est à tout moment menacé de voir sa démarche interrompue (dans le cas par exemple d'une rechute). Cette situation fait en sorte que plusieurs individus, qui auraient eu ainsi la possibilité de poursuivre une thérapie, ne la compléteront jamais étant donné un manquement à une condition imposée par le pénal.

Par ailleurs, la notion de confidentialitéa souvent été soulignée lors des groupes de discussion. En effet, selon les participants, le principe de confidentialité prôné par les intervenants en toxicomanie se heurte souvent aux besoins des instances judiciaires de vouloir surveiller adéquatement leur clientèle. Toutefois, les intervenants en toxicomanie affirment qu'ils peuvent consentir à livrer certaines informations avec le consentement explicite de leur client, telle l'attestation de présence, si cela est jugé nécessaire.

Finalement, il semble exister des contradictions majeures entre les messages de tolérance zéro prônés par la majorité des représentants des secteurs de la justice et de la sécurité publique et le message de réduction des méfaits entériné par certaines ressources pour toxicomanes.

Mais moi je dis toujours c'est comme si on avait un double message. C'est que le message officiel [du ministère] de la Sécurité publique c'est un peu tolérance zéro. Ne serait-ce qu'en exemple, quelqu'un qui est surpris en milieu carcéral en consommant de la drogue, des médicaments, avec une seringue, de l'héroïne, de la cocaine, tout ce que vous voulez, il y a rapport disciplinaire. Les gens qui font entrer une substance il peut y avoir une démarche entreprise via la cour pour que la personne soit accusée de trafic. Il y a cet aspect qui est très répressif pis en même temps... Je pense à des gens qui travaillent via le CLSC pis qui ont une approche réduction des méfaits. En même temps tu peux être l'intervenante qui est en train de faire du counseling, du support auprès de la personne alors moi je dis qu'il faut que le ministère [de la Sécurité publique] se positionne par rapport à la réduction des méfaits.

Bref, nous avons tenté d'exposer ici le plus fidèlement possible l'opinion des participants sur l'utilisation des pressions judiciaires dans le traitement de la toxicomanie en leur demandant de nous décrire le fonctionnement de telles mesures, leur apparente efficacité, les enjeux soulevés et les principales difficultés qu'ils rencontrent. 


\section{Discussion et pistes de solution}

L'arrestation, les procédures judiciaires et éventuellement l'expérience de la judiciarisation constituent pour certaines personnes une occasion de réflexion et de rupture avec un mode de vie mal endiguée. Elles représentent, pour bien des personnes qui en sont à leur premier contact avec l'appareil pénal, un choc; une occasion de changement. Par contre, il est reconnu que les milieux cœrcitifs ne favorisent pas la collaboration entre les contrevenants et les intervenants pénaux (Hirschel et Keny, 1990; Clément et Ray, 1991; Brochu et Schneeberger, à paraitre). Ceci incite donc à réfléchir sur les alternatives à l'incarcération pour l'infracteur toxicomane et à privilégier ainsi le traitement à des peines privatives de liberté.

L'intervention avec une clientèle référée par le système de justice est marquée par le sceau de l'ambiguité en raison de sa situation particulière, aux frontières du contrôle social et de l'aide thérapeutique. De quel côté se situe l'intervenant? S'agit-il d'un policier déguisé prêt à dénoncer tout manquement aux autorités carcérales ou d'un véritable thérapeute prêt à recevoir toutes confidences ?

L'espace clinique cœrcitif n'est pas un espace clinique libre. À tout le moins, il n'est pas libre de doutes dans l'esprit de la personne à aider. Dans ce contexte plusieurs questions se posent. Comment bien évaluer la motivation initiale du client ? Comment la faire progresser? Comment provoquer une alliance thérapeutique ? Comment la nourrir ? Comment donner le goût de poursuivre un cheminement thérapeutique au-delà de la période de contrainte judiciaire ?

Devant cette difficile situation, les intervenants en toxicomanie peuvent refuser les renvois du système judiciaire, prétextant à l'impossibilité de travailler dans un tel contexte; certains le font. Ils peuvent également y travailler en fermant toutes les voies de communication entre les deux systèmes. Toutefois, ce faisant, ils n'assurent plus l'indispensable communication qui permet de clarifier les attentes et les besoins de chacun et, surtout, de coordonner les efforts en vue de la réadaptation du contrevenant toxicomane. On isole alors la relation d'aide de l'infraction et de la sanction qui l'a fait naitre; on la dépossède d'une continuité structurante nécessaire à sa poursuite. Chacun agit alors pour défendre sa propre logique de fonctionnement plutôt que dans l'intérêt du client.

L'analyse des propos des intervenants qui ont participé à nos quatre groupes de discussion laisse croire qu'il y a beaucoup d'améliorations à apporter en matière d'intervention auprès d'une clientèle toxicomanejudiciarisée si l'on veut vraiment que cette alternative à l'incarcération 
subsiste. Les participants ont formulé plusieurs suggestions qui pourraient alimenter les réflexions et rendre plus efficace l'intervention en matière de toxicomanie chez une clientèle judiciarisée.

Tous les participants s'entendent sur la nécessité de mettre en place une meilleure concertation entre les différents partenaires impliqués dans la problématique toxicomanie-justice et la simplification des procédures à suivre lorsqu'un individu toxicomane est pris en charge par la justice. Une récente tournée de consultation orchestrée par le Comité permanent de lutte à la toxicomanie auprès d'un grand nombre d'intervenants québécois touchés par la problématique mettait d'ailleurs en évidence cette nécessité de concertation dans le champ de l'intervention en toxicomanie (Forget, 2000). Cette concertation des efforts, de même que cette simplification des procédures, peut passer par différentes initiatives. Selon les participants, quatre efforts sont à réaliser dans ce domaine.

Premièrement, il semble que plusieurs intervenants pénaux de première ligne souhaitent la mise en place de services d'évaluation et de référence en toxicomanie qui les aideraient dans l'orientation et, éventuellement, à la déjudiciarisation d'une partie de cette clientèle. Ce service devrait être facile d'accès (par ex. : numéro 1-800, télé-avertisseur, etc.) disponible en tout temps et indépendant d'un centre de traitement ou d'une instance judiciaire. En ce sens, on suggère de créer un poste de coordonnateur de l'évaluation et des références qui relèverait directement du ministère de la Santé et des Services sociaux. Plus tard, dans le processus judiciaire, une fois la sentence donnée, on suggère la mise en place d'activités de concertation inter-ministérielles (Santé et Services sociaux d'une part, et Justice et Sécurité publique, d'autre part) permettant l'évaluation et la référence de cas par des experts du domaine de la délinquance et de la toxicomanie. En effet, on a trop souvent l'impression que le travail isolé de ces experts ne permet pas une évaluation adéquate de cette double problématique et que le manque de communication entre eux a pour effet de laisser une trop grande place à la manipulation des intervenants par des contrevenants plus soucieux de leur confort que de leur cheminement psychologique.

Deuxièmement, il faut développer une plus grande expertise en matière de toxicomanie et de délinquance. L'acquisition d'expertise passe par des stratégies de formation/information des instances décisionnelles (par ex. : juges, commissaires aux libérations conditionnelles, etc.) et des professionnels en place (intervenants des divers réseaux impliqués) qui jouent des rôles déterminants dans les trajectoires pénales et de réadaptation des 
contrevenants toxicomanes. Malheureusement, ces personnes ne sont pas toujours bien au fait des besoins de cette clientèle, des ressources disponibles pour y répondre et des conditions optimales de renvoi.

Troisièmement, le système de justice a la capacité de créer une pression efficace (et jusqu'à un certain point justifiée) favorisant ainsi l'adhésion à une démarche de changement. Toutefois, ces pressions ne devraient être utilisées que lorsque ces personnes ne sont pas déjà rejointes par des services de traitement. De plus, elles ne devraient viser que les personnes qui éprouvent réellement des problèmes de toxicomanie (et non pas les simples consommateurs ou les personnes accusées de trafic de drogues). Bien plus, avant d'être référé à un centre de traitement, le client devrait pouvoir être informé des grandes lignes d'une démarche de réadaptation, des conditions de sa réalisation, de la nature des services offerts par différents centres de traitement, du fonctionnement de ces ressources, et des attentes des thérapeutes (par ex. : dévoilement de soi). Ces réflexions mettent donc l'accent sur l'importance de présenter une position claire et sans ambiguitéaux clients et aux intervenants à l'égard des attentes des différents partis (représentants de la justice et des ressources pour toxicomanes) et face à leur rôle à jouer vis-à-vis de la clientèle et ce, dès le début de la prise en charge. Selon le protocole d'entente entre le ministère de la Santé et des Services sociaux et le ministère de la Sécurité publique, ce dernier est responsable de l'évaluation de sa clientèle toxicomane, mais peut faire largement appel à l'expertise des intervenants du ministère de la Santé et des Services sociaux. Toutefois, en cas de non-judiciarisation ou de déjudiciarisation, cette responsabilité incombe au ministère de la Santé et des Services sociaux (Fédération québécoise des CRPAT, 1993). La clarification des rôles doit insister sur le fait que la notion de sécurité doit être réservée à ses experts, c'est-à-dire les intervenants judiciaires et pénaux. La clarification des positions adoptées touche en particulier le concept de rechute et les conséquences qui peuvent y être associées. Les intervenants du réseau judiciaire et pénal doivent être conscients de la signification de la rechute pour un intervenant en toxicomanie et du matériel qu'il peut y puiser si les contraintes des autorités judiciaires ne l'empêchent pas d'y avoir accès.

Finalement, la réinsertion des personnes toxicomanes qui sont condamnées à une peine de prison semble être le problème majeur. En effet, lorsque ces personnes ne reçoivent aucun traitement durant leur période de détention, elles sont généralement peu motivées à entreprendre une telle démarche une fois libérées (à moins que ce ne soit une condition spéci- 
fique de leur mise en liberté). Si ces personnes peuvent bénéficier de services de traitement durant leur période d'incarcération, il y a fort à parier qu'elles ne pourront jouir de services de réinsertion directement reliés aux traitements reçus durant leur incarcération.

En somme, bien qu'un certain nombre d'ententes toxico-justice régionales aient été paraphées au cours des dernières années, l'analyse de la situation actuelle telle que dépeinte par les participants laisse l'impression que, depuis la signature du protocole d'entente entre le ministère de la Sécurité publique et le ministère de la Santé et des Services sociaux en 1989, trop peu de progrès ont été réalisés en ce domaine au cours des dix dernières années laissant ainsi la majorité des intervenants impliqués insatisfaits de la situation actuelle.

\section{Conclusion}

Le traitement du contrevenant toxicomane constitue certes une meilleure solution que son emprisonnement. Nous le savons bien, la prison peut représenter un lieu de solidification d'une trajectoire déviante; l'école du crime dit-on ! Certains détenus y trouvent la confirmation de leur statut; ils y acquièrent des gallons; ils y développent de nouvelles habiletés.

L'étude présentée a tenté de mettre en relief les principales difficultés que rencontrent les intervenants pénaux et sociaux dans leurs efforts de réadaptation des contrevenants toxicomanes. Certes, une des limites de l'étude est le faible nombre de participants interrogés et l'absence d'intervenants provenant de certains domaines (juges, procureurs). Par contre, une étude conduite en 1995 auprès d'un nombre beaucoup plus important d'intervenants parvenait, à l'époque, à des conclusions similaires à celles qui viennent d'être présentées (Schneeberger et Brochu, 1999).

Ceci dit, il faut tout de même convenir que l'arrimage entre le réseau judiciaire ou pénal et celui de la santé et des services sociaux ne se fait pas sans difficultés. Les premières difficultés se manifestent dès le processus d'accueil en traitement alors que dans bien des cas les thérapeutes doutent de la réelle volonté de la personne référée par le système judiciaire à vouloir vraiment entreprendre une démarche de changement. En effet, bon nombre de thérapeutes croient que l'intérêt immédiat des contrevenants (à savoir rendre son cheminement pénal le moins 
pénible et le plus court possible) ne concorde pas nécessairement avec des objectifs de réadaptation.

En effet, dans bien des cas, la demande d'aide d'un contrevenant a été plus ou moins subtilement initiée par le système de justice. Dans ces circonstances, il n'est pas surprenant d'apprendre que les intervenants pénaux au Québec se plaignent de leurs grandes difficultés à évaluer la réelle motivation des personnes rencontrées dans le contexte de contrôle dans lequel ils travaillent. Pourtant, une véritable démarche thérapeutique doit se faire dans un environnement qui préserve un espace d'engagement réel dans le processus de changement. Ce n'est qu'à cette condition que pourra prendre forme l'alliance thérapeutique qui constitue la base du traitement. Toutefois, lorsque le contexte pénal encadre le processus de réadaptation, il risque d'empoisonner l'espace thérapeutique par ses nombreuses contraintes administratives (par ex. : respect des conditions de non consommation; rédaction de rapports). Ce faisant, il risque d'assimiler le thérapeute à l'autorité judiciaire.

Le traitement doit donc s'effectuer sous un constant regard critique. La priorité qui est placée sur la protection de la société et les impératifs administratifs ne doivent pas contaminer la nature du traitement et du processus de réadaptation qu'il est censé initier.

Ainsi, l'objectif naturel du processus de réadaptation que constitue le bien-être psychologique du client ne doit pas être remplacé par la volonté d'atteindre un niveau de récidive zéro. Comme nous l'avons vu, pour le réseau judiciaire et pénal, l'indicateur de récidive par excellence en matière de toxicomanie est la rechute. De nombreuses énergies sont consacrées par les intervenants pénaux pour mesurer cette rechute, la détecter et éventuellement la prévenir. Pourtant, dans le champ de l'intervention en toxicomanie, la rechute constitue un matériel de premier plan. C'est souvent à travers elle que le travail thérapeutique se réalise et que le client progresse. Pour plusieurs personnes, la toxicomanie consiste en une réponse (inadaptée dans beaucoup de cas) à une série de difficultés profondes. Le traitement de la toxicomanie consiste, en quelque sorte, en l'apprentissage de stratégies d'adaptation alternatives. La consommation problématique ne constitue qu'un symptôme de problèmes plus importants. Plusieurs clients expérimentent pour la première fois depuis de nombreuses années la vie sans drogue. Il est important que cette expérience puisse être vécue comme une réussite en favorisant le désir de poursuivre dans cette voie, avenue qu'on ne peut atteindre en gérant la rechute comme un échec de la démarche. La 
rechute constitue donc une indication d'un besoin de traitement et non de l'inverse (expulsion). L'expulsion devrait plutôt être appuyée sur un échec thérapeutique qui n'équivaut pas uniquement à une rechute, sinon elle est éthiquement inacceptable et socialement dangereuse.

En somme, la relation client/thérapeute à l'intérieur d'un cadre cœrcitif peut rapidement devenir un jeu de dupes, obligeant l'un à fournir une preuve des progrès réalisés et l'autre à devenir de plus en plus sceptique face aux manifestations de ces progrès. Il faut également bien être conscient que lorsque l'intervenant s'attarde à faire appliquer des règles rigides imposées par le réseau pénal, ce dernier n'est pas en position pour utiliser de façon adéquate la période de contact avec le client pour établir la relation thérapeutique favorisant la réadaptation en raison du court laps de temps dont il dispose.

\section{Références}

Anglin, M. D., Brecht, M. L. et Maddahian, E. 1990. «Pre Treatment Characteristics and Treatment Performance of Legally Cœrced Versus Voluntary Methadone Maintenance Admissions» Criminology 23 : 1011-1027.

Anglin, M. D. et Hser, Y. I. 1990. « Treatment of Drug Abuse », Pp. 393-460 in Drugs and Crime, sous la direction de M. Tonry et J. Q. Wilson. Chicago : The University of Chicago Press.

Ball, J. C., Rosen, L., FlueCK, J. A. et Nurco, D. N. 1981. «The Criminality of Heroin Addicts : When Addicted and When Off Opiates », Pp. 39-65 in The Drug-Crime Connection, sous la direction de J. A. Inciardi. Beverly-Hills, CA : Sage.

Beaudet, N., Brochu, S., Bergeron, J. et Landry, M. 1995. Persistance en traitement et impact du processus de réadaptation des personnes toxicomanes judiciarisées admises à Domrémy-Montréal. Rapport no 5, Université de Montréal : Centre international de criminologie comparée.

Brecht, M. L., ANGLin, M. D. et WANG, J. C. 1993. « Treatment Effectiveness for Legally Coerced Versus Voluntary Methadone Maintenance Clients » American Journal of Drug and Alcobol Abuse 19 (1) : 89-106.

BRochu, S. 1995. Drogue et criminalité : Une relation complexe, Montréal : Les Presses de l'Université de Montréal.

Brochu, S., Bergeron, J., Brunelle, N., Cournoyer, L.G., Landry, M. et Tremblay, J. 1999. Comprébension de l'impact d'un traitement pour personnes toxicomanes-judiciarisées à partir d'un modèle tripartite : l'intervention, le client et son environnement, Demande de subvention déposée au Conseil québécois de la recherche sociale (CQRS).

Brochu, S., Bergeron, J., Landry, M. et Germain, M. 1999. Interventions adaptées aux personnes toxicomanes judiciarisées, Montréal : Centre international de criminologie comparée. 
Brochu, S., Cournoyer, L-G., Motiuk, L. et Pernanen, K. (sous presse). « Drugs, Alcohol and Crime: Patterns among Canadian Federal Inmates » Bulletin of Narcotics.

BROCHU, S. et SCHNEEBERGER, P. 1997. Services à offrir aux personnes présentant une double problématique toxicomanie/délinquance, Montréal : Les cahiers de recherche criminologiques.

Brochu, S. et SCHneEberger, P. (à paraitre). « Le traitement de la toxicomanie en contexte carcéral : politiques et éthique» in L'usage des drogues et la toxicomanie III, sous la direction de P. Brisson.

CLÉMENT, M. et RAY, D. 1991. « Les intervenants extérieurs au milieu carcéral : les limites de leur intervention » Bulletin Liaison CNDT 17 : 45-48.

De LeON, G. 1988. "Legal Pressure in Therapeutic Communities » The Journal of Drug Issues 18 (4) : 625-640.

Fédération québécoise des CRPAT 1993. Les collaborations entre les centres de réadaptation pour personnes alcooliques et autres personnes toxicomanes et les milieux judiciaires : position provinciale, Montréal : Fédération québécoise des centres de réadaptation pour personnes alcooliques et autres toxicomanes.

FORGET, J. 2000. Éléments majeurs issus de l'analyse des données recueillies au cours des phases I et II de la consultation, Présentation dans le cadre de la consultation 1999-2000 du Comité permanent de lutte à la toxicomanie, Montréal, 21 juin 2000.

Hirschel, J.D. et KenY, J.R. 1990. «Outpatient Treatment for Substance Abusing Offenders » Journal of Offender, Counseling, Services and Rebabilitation $15: 111-130$.

Hubbard, R. L., Collins, J. J., Rachal, J. V. et Cavanaugh, E. R. 1988. « The Criminal Justice Client in Drug Abuse Treatment » NIDA Research Monograph Series 86 : 57-80. Rockville, MD : National Institute on Drug Abuse.

Mumola, C.J. 1998. Substance Abuse and Treatment, State and Federal Prisoners, 1997, Washington : Bureau of Justice Statistics.

SCHNEEBERGER, P. et Brochu, S. 1999. "L'intervention auprès des toxicomanes judiciarisés : les intervenants et intervenantes se prononcent » Revue Canadienne de Santé Mentale Communautaire 18 (1) : 181-197.

SChwartz, J.R. et SCHwartz, L.P. 1998. « The Drug Court: A New Strategy for Drug Use Prevention » Obstetrics and Gynecology Clinics of North Amercica 25 (1) : 255-268.

Simpson, D. D., Joe, L. J., Lehman, W. E. K. et Sells, S. B. 1986. « Addiction Careers: Etiology, Treatment, and 12-Years Follow-Up Outcomes » Journal of Drug Issues 16 (1) : 107-121.

STEER, R. A. 1980. "Psychosocial Correlates of Retention in Methadone Maintenance » The International Journal of the Addictions 15 (7) : 1003-1009.

TORRES, S. 1996. «Should Corrections Treat or Punish Substance-Abusing Criminals » Federal Probation 60 (3) : 18-23.

Van Stelle, K. R., Mauser, E. et Moberg, D. P. 1994. «Recidivism to the Criminal Justice System of Substance-Abusing Offenders Diverted Into Treatment » Crime and Delinquency 40 (2) : 175-196. 